\title{
MECHANISMS OF MYOCARDIAL PROTECTION BY ADENOSINE-SUPPLEMENTED CARDIOPLEGIC SOLUTION: MYOFILAMENT AND METABOLIC RESPONSES
}

Bernard G. Fogelson, $\mathrm{MD}^{\mathrm{c}}$

Sammy I. Nawas, $\mathrm{MD}^{\mathrm{c}}$

William R. Law, $\mathrm{PhD}^{\mathrm{a}, \mathrm{b}, \mathrm{c}}$
Objective: Adenosine supplementation of cardioplegic solutions in cardiac operations improves postarrest myocardial recovery after cardioplegic arrest and reperfusion; however, the mechanism of the action of adenosine remains unknown. We tested the hypotheses that adenosinesupplemented cardioplegic solution improves myofibrillar protein cooperative interaction and increases myocardial anaerobic glycolysis. Methods: The hearts of male Sprague-Dawley rats were randomized to undergo 120 minutes of cardioplegic arrest with 1 of 3 cardioplegic solutions: (1) St Thomas' Hospital No. 2 cardioplegic solution (St Thomas group), (2) St Thomas' Hospital No. 2 cardioplegic solution plus adenosine (100 $\mu \mathrm{mol} / \mathrm{L})$ (adenosine group), and (3) St Thomas' Hospital No. 2 cardioplegic solution plus adenosine $(100 \mu \mathrm{mol} / \mathrm{L})$ plus the nonspecific adenosine receptor antagonist 8-p-sulfophenyltheophylline $(50 \mu \mathrm{mol} / \mathrm{L})$ (sulfophenyltheophylline group). A fourth group of hearts underwent no cardioplegic arrest. Results: Systolic and diastolic functional recovery was improved in the adenosine group compared with that in the other two groups, independent of coronary flow. Adenosine supplementation of cardioplegic solution prevented the decrease in myofibrillar protein cooperative interaction seen after cardioplegic arrest and reperfusion (St Thomas and sulfophenyltheophylline groups). Adenosine-supplemented cardioplegic solution also caused significantly increased anaerobic glycolysis during cardioplegic arrest. These responses were blocked in the sulfophenyltheophylline group. Conclusions: The changes in myocardial glycolytic activity and myofilament cooperativity coincided with functional recovery in the three cardioplegia groups and may represent mechanisms underlying protection with adenosine-supplemented cardioplegic solution. (J Thorac Cardiovasc Surg 2000;119:601-9)
$\mathrm{M}$ yocardial depression after cardiac surgical procedures is a common clinical occurrence. Studies have shown that up to $90 \%$ of patients undergoing coro-

From the Research Service, ${ }^{\mathrm{a}}$ Westside Veterans Administration Medical Center, and the Departments of Physiology and Biophysics $^{\mathrm{b}}$ and Surgery, ${ }^{\mathrm{c}}$ University of Illinois College of Medicine at Chicago, Chicago, Ill.

Supported by a Veterans Administration Merit Review grant, a Living Institutes for Surgical Studies (LISS) grant, and National Institutes of Health grant GM 48219 (Dr Law). Dr Nawas was an Eleanor B. Pillsbury Fellow.

Received for publication April 12, 1999; revisions requested Sept 2, 1999; revisions received Oct 5, 1999; accepted for publication Oct 22, 1999

Address for reprints: William R. Law, PhD, Associate Professor, University of Illinois at Chicago, Department of Physiology and Biophysics (M/C 901), 835 S Wolcott, Chicago, IL 60612 (E-mail: wrlaw@uic.edu).

12/1/104041

doi:10.1067/mtc.2000.104041 nary artery bypass grafting have a decrease in ejection fraction or cardiac index in the immediate postoperative period as compared with respective preoperative values. ${ }^{1}$ Although there have been changes in cardioplegic solution composition and changes in the methods of delivering the solution over the past 20 years, this is still a significant cause of morbidity and mortality in the postoperative period.

Adenosine was introduced as an additive to cardioplegic solution in 1980 by Foker, Einziz, and Wang. ${ }^{2}$ Since that time numerous studies have detailed the improved myocardial protection attributable to adenosine supplementation of cardioplegic solution. ${ }^{3-5}$ However, the fundamental mechanism or mechanisms by which adenosine augments myocardial protection during cardioplegic arrest are incompletely understood. Silverman and associates ${ }^{6}$ theorized that adenosine provides ultrastructural substrate for adenosine triphos- 
phate (ATP) repletion and thus attenuates the depression of high-energy phosphates after cardioplegic arrest and reperfusion. However, in 1994 Hudspeth and coworkers $^{3}$ reported that the actions of adenosine in cardioplegia are receptor mediated. Much work has been done to further our understanding of the mechanisms of the receptor-mediated actions of adenosine in normothermic perfused and ischemic hearts, ${ }^{7-13}$ but it would be presumptive to extrapolate these findings to the cardioplegia setting. Indeed, the response of myofilaments to cardioplegic arrest differs significantly from the response seen in warm ischemic injury. ${ }^{14}$ The hypothermia alone, typical of cardioplegic arrest, can alter enzyme kinetics and receptor binding characteristics.

Earlier studies in our laboratory demonstrated that cardioplegic arrest induces changes that are reflected in altered biochemical interactions in the myofibrillar contractile apparatus. ${ }^{14}$ Specifically, the cooperative interaction of the actin-myosin $\mathrm{Mg}^{+2}$ ATPase enzyme activity is decreased, which would contribute to depressed systolic and diastolic functional recovery. We therefore tested the hypothesis that adenosine, via a receptor-mediated process, attenuates or prevents the decrease in the myofibrillar protein cooperative interaction seen after cardioplegic arrest and reperfusion.

Another potential mechanism by which adenosine might protect the arrested heart is via potentiation of cardiac glycolysis. Glycolysis is vital to protecting cardiac function during warm ischemia,${ }^{8}$ conditions of reduced oxygen supply in relation to demand, ${ }^{10}$ and during cardioplegic arrest. ${ }^{15}$ Adenosine can potentiate cardiac glycolysis, ${ }^{11-13}$ and this response is important under normothermic conditions. ${ }^{8,10}$ Again, however, these results cannot be extrapolated easily to the setting of cold cardioplegic arrest, a setting of significantly reduced biochemical processes and energy requirements. Therefore we thought it important to determine whether adenosine-mediated potentiation of glycolysis played a role in the ability of adenosine to protect the heart during cardioplegic arrest. To this end, we tested the hypothesis that increased myocardial glycolysis is associated with the salutary effects of adenosine in cardioplegia.

\section{Methods}

The animals used in the experiments reported herein received humane care in compliance with the "Guide for the Care and Use of Laboratory Animals," prepared by the Institute of Laboratory Animal Resources and published by the National Institutes of Health (NIH publication 86-23, revised 1985).
Isolated heart protocol. Male Sprague-Dawley rats (373.1 $\pm 8.5 \mathrm{gm})$ were randomized to 1 of 4 experimental groups (see "Experimental groups" section). Rats were anesthetized with a $50 \mathrm{mg} / \mathrm{kg}$ dose of intraperitoneal pentobarbital sodium. After adequate anesthesia was ensured by absence of the deep pain reflex to interdigital pinch, a laparotomy was performed. Two minutes after injection of 200 units of heparin into the inferior vena cava the chest was rapidly opened and the heart was removed and placed in ice-cold Krebs Ringer bicarbonate buffer. Extraneous tissue was dissected free, the left and right ventricles vented with a 20 -gauge needle, and the aortic root rapidly cannulated. The cannula was then connected to a non-recirculating, temperature-controlled, isolated heart perfusion apparatus. The heart was perfused in an aortic retrograde (coronary antegrade) fashion at a constant pressure of $80 \mathrm{~mm} \mathrm{Hg}$ with warm $\left(37^{\circ} \mathrm{C}\right)$ oxygenated $(95.5 \%$ oxygen $/ 4.5 \%$ carbon dioxide) modified Krebs Ringer bicarbonate buffer containing $100 \mathrm{mmol} / \mathrm{L} \mathrm{NaCl}, 4.74 \mathrm{mmol} / \mathrm{L}$ $\mathrm{KCl}, 1.12 \mathrm{mmol} / \mathrm{L} \mathrm{CaCl}_{2}, 1.18 \mathrm{mmol} / \mathrm{L} \mathrm{MgSO}_{4}, 25 \mathrm{mmol} / \mathrm{L}$ $\mathrm{NaHCO}_{3}$, and $1.18 \mathrm{mmol} / \mathrm{L} \mathrm{KH}_{2} \mathrm{PO}_{4}$ and modified with the addition of $11.4 \mathrm{mmol} / \mathrm{L}$ glucose, $4.92 \mathrm{mmol} / \mathrm{L}$ pyruvate, and $5.38 \mathrm{mmol} / \mathrm{L}$ fumarate. After gas equilibration, the $\mathrm{pH}$ was titrated to 7.4 with $0.1 \mathrm{~N} \mathrm{HCl}$ and $\mathrm{KOH}$. Hearts were paced at 300 beats/min with bipolar silver electrodes embedded in the right atrium at $125 \%$ of the minimal voltage required to pace the heart at a duration of $6 \mathrm{~ms}$ with a Grass SD9 stimulator (Grass Instruments, Quincy, Mass). A latex balloon surrounding a microtransducer-tipped Millar catheter with lumen (Millar Instruments, Houston, Tex) was inserted into the left ventricle via the mitral valve. The Millar catheter was connected to an AT class computer (Dell, Inc, Austin, Tex) and data were recorded using the CODAS data acquisition system (DATAQ, Inc, Akron, Ohio) at a digital conversion frequency of 250 samples per second per channel. The latex balloon was inflated and deflated multiple times to adequately seat the balloon in the left ventricle. Sufficient volume was then kept in the balloon to maintain a stable left ventricular end-diastolic pressure (LVEDP) of 0 to $2 \mathrm{~mm} \mathrm{Hg}$. The preparation was considered stable if the diastolic and systolic pressures remained constant (diastolic pressure variation $<2 \mathrm{~mm}$ $\mathrm{Hg}$; systolic variation $<5 \mathrm{~mm} \mathrm{Hg}$ ) for 15 minutes. Hearts were excluded if the developed pressure was less than $60 \mathrm{~mm}$ $\mathrm{Hg}$ or if they exhibited ventricular arrhythmias or were unable to be paced.

Experimental protocol. After a 15-minute stabilization period, measurements of left ventricular pressure (LVP) were recorded and variably loaded pressure-volume (Starling) curves generated via incremental $(5 \mu \mathrm{L})$ volume unloading by means of a Harvard pump (model 55-2226; Harvard Apparatus Co, South Natick, Mass). Volume was slowly added into the left ventricular balloon until an end-diastolic pressure of $20 \mathrm{~mm} \mathrm{Hg}$ was reached. The volume was then removed from the balloon in $5-\mu \mathrm{L}$ decrements until an enddiastolic pressure of $0 \mathrm{~mm} \mathrm{Hg}$ or less was reached. After the withdrawal of each successive $5-\mu \mathrm{L}$ volume, the ventricle was allowed to stabilize for 15 seconds and LVP tracings 
were recorded. This procedure was then repeated starting again at an end-diastolic pressure of $20 \mathrm{~mm} \mathrm{Hg}$. In addition to these readings, coronary flow was measured and effluent samples for glucose and lactate measurement were obtained and later analyzed (YSI model 2300; Yellow Springs, Ohio). Then $3 \mathrm{~mL}$ of one of the three cardioplegic solutions (see "Experimental groups" section) at $4^{\circ} \mathrm{C}$ was infused into the aortic cannula to arrest the heart. Infusion pressure was monitored to prevent the infusion pressure from rising above 60 $\mathrm{mm} \mathrm{Hg}$. Thereafter $1 \mathrm{~mL}$ of cardioplegic solution was infused every 15 minutes for a total arrest time of 120 minutes. Glucose and lactate effluent samples were obtained during infusion of the cardioplegic solution at 30,60, and 90 minutes of cardioplegic arrest. At the end of the 120-minute cardioplegic arrest period, hearts were reperfused with warm oxygenated Krebs Ringer bicarbonate buffer (as used during the stabilization period) for 1 hour. At 30 and 60 minutes of reperfusion, hemodynamic measurements were obtained in the same manner as described previously, and coronary flow measurements and effluent samples for glucose and lactate levels were collected. At the end of 60 minutes of reperfusion, hearts were freeze-clamped with Dry Ice and stored at $-70^{\circ} \mathrm{C}$ for later biochemical analysis.

Experimental groups. All cardioplegic solutions were oxygenated with $95.5 \%$ oxygen $/ 4.5 \%$ carbon dioxide and maintained at a temperature of $4^{\circ} \mathrm{C}$. The first group (ST\#2) received St Thomas' Hospital No. 2 cardioplegic solution. This solution comprised $110 \mathrm{mmol} / \mathrm{L} \mathrm{NaCL}, 16 \mathrm{mmol} / \mathrm{L} \mathrm{KCl}$, $1.2 \mathrm{mmol} / \mathrm{L} \mathrm{CaCl}_{2}, 16 \mathrm{mmol} / \mathrm{L} \mathrm{MgCl}_{2}$, and $10 \mathrm{mmol} / \mathrm{L}$ $\mathrm{Na}_{2} \mathrm{HCO}_{3}$. To determine the effects of exogenous adenosine supplementation on myocardial protection, the second group (ADO) received a cardioplegic solution that consisted of $\mathrm{St}$ Thomas' Hospital No. 2 solution supplemented with adenosine (Sigma, St Louis, Mo) to a final concentration of 100 $\mu \mathrm{mol} / \mathrm{L}$. The third group (SPT) received a cardioplegic solution that consisted of St Thomas' Hospital No. 2 solution plus adenosine $(100 \mu \mathrm{mol} / \mathrm{L})$ plus the nonspecific adenosine receptor blocker 8-p-sulfophenyltheophylline $(50 \mu \mathrm{mol} / \mathrm{L}$; Research Biochemicals International, Natick, Mass). The purpose of the third group was to determine which effects of adenosine during cardioplegic arrest were adenosine-receptor mediated. The doses of adenosine and 8-p-sulfophenyltheophylline were based on doses used in other studies. ${ }^{9}$ A separate fourth group of hearts (No CPL) did not undergo cardioplegic arrest. Instead, these hearts were perfused with warm, oxygenated Krebs Ringer bicarbonate buffer for 60 minutes after baseline readings were obtained (total perfusion time, 90 minutes). These hearts were used for comparison of functional and biochemical changes associated with the total amount of time all hearts underwent beating, warm, oxygenated perfusion. Animals were randomly assigned to one of the experimental groups until a minimum of 6 rats in each group was attained. The requirement of a minimum of 6 rats per group was based on the variance of myofibrillar ATPase data being measured, applied to power analysis with $\alpha$ set at .8 and $P<.05$ required for statistical significance. Random assignment of rats to these groups resulted in 7 rats in the ST\#2 group, 6 rats in the ADO group, 8 rats in the SPT group, and 6 rats in the No CPL group (total 27 rats).

\section{Functional analysis}

Systolic function. To assess systolic function across time and between the groups, systolic pressure was obtained at 2 and $10 \mathrm{~mm} \mathrm{Hg}$ preload pressure. The maximal value of the first derivative of pressure over time (peak $[+] \mathrm{dP} / \mathrm{dt}$ ) was determined at an LVEDP of $10 \mathrm{~mm} \mathrm{Hg}$.

Diastolic function: compliance measurements. Diastolic compliance was determined from data defining the end-diastolic pressure-volume relationship (EDPVR). The LVEDP for each cardiac cycle was defined as the point on the LVP tracing that corresponded to the first $(+) \mathrm{dP} / \mathrm{dt}$ value greater than $50 \mathrm{~mm} \mathrm{Hg} / \mathrm{s}$. This value of LVEDP corresponded to the point on the LVP tracing immediately preceding the rapid upstroke of systole. LVEDP values were averaged for 4 cardiac cycles at each preload volume. The values for the EDPVR were fit to the following equation: $L V P=A+B$. $\left(\exp ^{\mathrm{C} \cdot \mathrm{Vx}}-1\right)$, where $\mathrm{A}$ is the $\mathrm{Y}$ intercept and $\mathrm{B}$ and $\mathrm{C}$ are modifiers of the exponential curvature of the EDPVR. ${ }^{16}$ Volume strain was calculated by normalizing volume for the equilibrium volume $\left(\mathrm{V}_{\mathrm{o}}\right)$, which is the volume at $\mathrm{LVP}=0$. Volume strain was calculated by the equation $\mathrm{V}_{\mathrm{n}}=\left(\mathrm{V}-\mathrm{V}_{\mathrm{o}}\right) / \mathrm{V}_{\mathrm{o}}$. To determine compliance, volume strain was plotted against stress (pressure millimeters of mercury). This relationship was fitted to the equation $\mathrm{Y}=\mathrm{A} \cdot\left(\exp ^{\mathrm{B} \cdot \mathrm{Vn}}-1\right)$, where $\mathrm{A}$ and $\mathrm{B}$ are modifiers of the exponential curvature of the stress/ strain relationship. Changes in regression coefficients from baseline values were analyzed for significant alterations in compliance after cardioplegic arrest.

Myofibrillar preparations. Left ventricular myofibrillar proteins were isolated by use of a modification of the general method of Pagani and Solaro, as previously reported. ${ }^{14}$ All isolation procedures were done at $0^{\circ} \mathrm{C}$ to $4^{\circ} \mathrm{C}$ and all solutions contained leupeptin $(0.5 \mathrm{mg} / \mathrm{mL})$, pepstatin A $(0.5$ $\mathrm{mg} / \mathrm{mL})$, and phenylmethylsulfonyl fluoride $(0.2 \mathrm{mmol} / \mathrm{L})$ to inhibit proteolytic enzymes. Ventricles were minced in a solution containing $10 \mathrm{mmol} / \mathrm{L} \mathrm{EGTA},{ }^{*} 2 \mathrm{mmol} / \mathrm{L} \mathrm{MgATP}, 6$ $\mathrm{mmol} / \mathrm{L}$ phosphocreatine, $1.65 \mathrm{mmol} / \mathrm{L} \mathrm{Mg}^{2+}, 36 \mathrm{mmol} / \mathrm{L}$ $\mathrm{KCl}$, and $60 \mathrm{mmol} / \mathrm{L}$ imidazole, homogenized, and centrifuged. The pellet was resuspended in the solution described (except EGTA was reduced to $0.1 \mathrm{mmol} / \mathrm{L}$ ) and centrifuged. To remove membrane contaminates, the pellet was resuspended in standard buffer $(60 \mathrm{mmol} / \mathrm{L} \mathrm{KCl}, 2.0 \mathrm{mmol} / \mathrm{L}$ $\mathrm{MgCl}_{2}, 30 \mathrm{mmol} / \mathrm{L}$ imidazole; $\mathrm{pH}$ 7.0) containing $1 \%$ Triton $\mathrm{X}-100$ ( $\mathrm{vol} / \mathrm{vol})$, left on ice for 20 minutes, and centrifuged. The pellet was washed by repeating the resuspension in standard buffer and the centrifugation steps 3 times. Myofilament protein concentration was determined by means of the modified Lowry protein assay described by Pagani and Solaro, as previously reported. ${ }^{14}$

ATPase measurements. $\mathrm{Ca}^{2+}$-dependent myofibrillar ATPase activities were measured as described previously. ${ }^{14}$ In brief, myofibrillar $\mathrm{Ca}^{2+}$-dependent $\mathrm{MgATPase}$ activity was

*Ethyleneglycoltetraacetic acid. 


\section{Systolic Pressures}
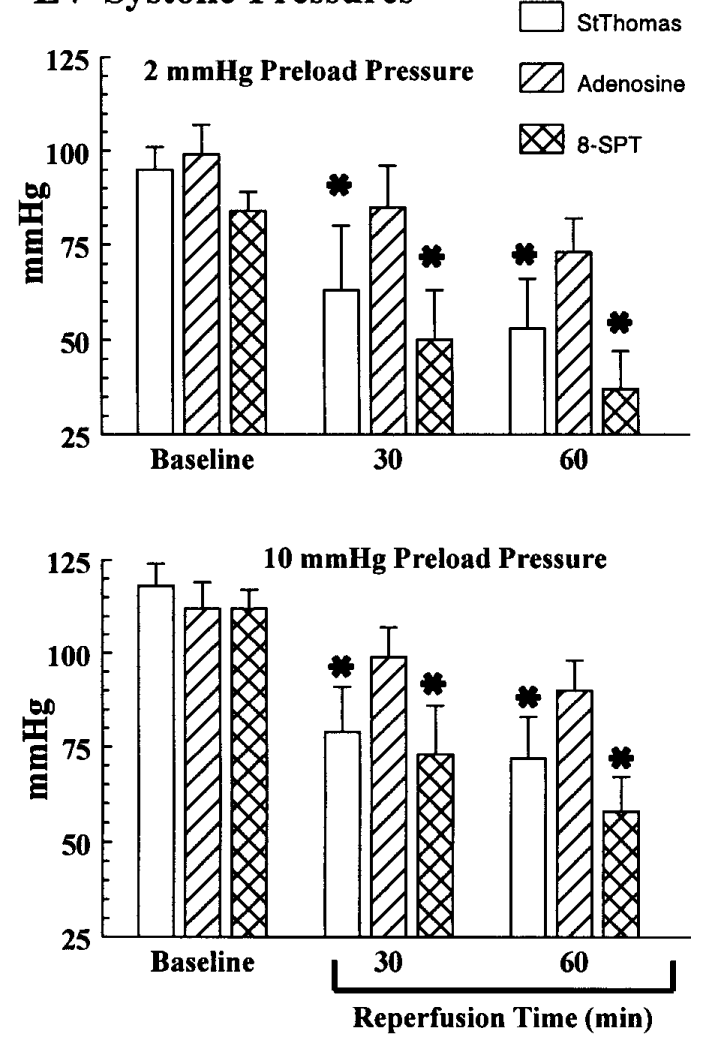

Fig 1. Left ventricular systolic pressures at preload pressures of 2 and $10 \mathrm{~mm} \mathrm{Hg}$. At $2 \mathrm{~mm} \mathrm{Hg}$ systolic pressure preload (top panel), the systolic pressure generated in the ST\#2 group $(n=7)$ decreased significantly from baseline at 30 and 60 minutes of reperfusion. In the adenosine-supplemented group (ADO; $n=6$ ) there was no significant decrease in systolic pressure generated. However, in the adenosine-receptorblocked group (SPT; $n=7$ ) there was a significant decrease in systolic pressure generation at both 30 and 60 minutes of reperfusion. Similar results were obtained at $10 \mathrm{~mm} \mathrm{Hg}$ preload pressure (bottom panel). ${ }^{*} P<.05$ compared with baseline.

measured in a reaction solution containing 0.2 to $0.5 \mathrm{mg} / \mathrm{mL}$ myofibrillar protein, $66 \mathrm{mmol} / \mathrm{L} \mathrm{KCl}, 2 \mathrm{mmol} / \mathrm{L} \mathrm{Mg}^{2+}, 60$ mmol/L imidazole (pH 7.0), $1 \mathrm{mmol} / \mathrm{L}$ EGTA, and $5 \mathrm{mmol} / \mathrm{L}$ $\mathrm{MgATP}^{2-}$ and varied $\mathrm{Ca}^{2+}$ concentrations (pCa 8.0 to $4.5 ; \mathrm{pCa}$ $=-\log \left[\mathrm{Ca}^{2+}\right]$ ). The ATPase reaction was initiated with the addition of ATP and stopped after 10 minutes with $10 \%$ cold trichloroacetic acid. The reaction mixture was centrifuged at $4^{\circ} \mathrm{C}$ to pellet the protein. Inorganic phosphate produced by ATP hydrolysis was measured in the supernatant fraction by the method of Carter and Karl, as previously reported. ${ }^{14}$ ATPase activities were reported as nanomoles inorganic phosphate produced per milligram protein per minute.

Statistics. All values are presented as means \pm SEM. After homogeneity of variance was assured, group and/or time differences were determined by 1-way and 2-way analyses of

\section{Compliance}

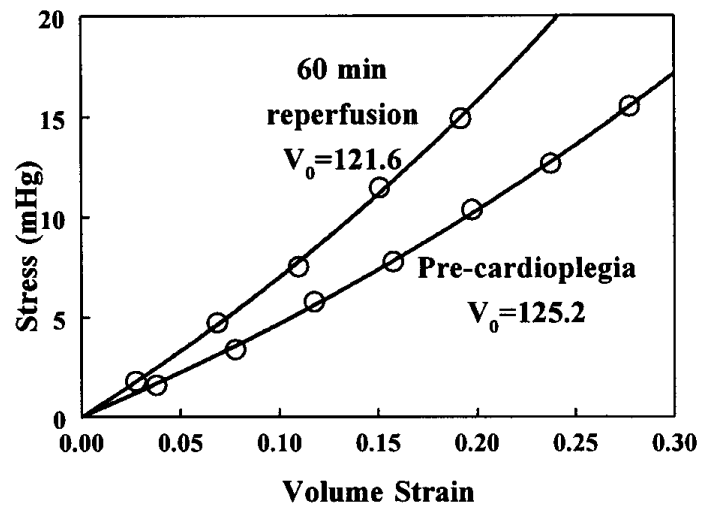

Fig 2. Compliance (stress/strain) curves for a representative heart in the ST\#2 group. Data points (open circles) were fit to the monoexponential equation: Stress $=A \cdot\left(\exp ^{B} \cdot V_{-1}\right)$. Before cardioplegic arrest the values of regression coefficients A and B were 22.817 and 1.869, respectively. After 60 minutes of reperfusion, coefficients $\mathrm{A}$ and $\mathrm{B}$ increased to 27.914 and 2.237, respectively. These changes in A and B translate to a decrease in compliance or a shift of the compliance curves up and to the left. $V_{o}$, Equilibrium volume.

variance as appropriate (see following paragraph). The number of samples per group was as indicated previously, with exceptions indicated in the text and legends where measurements were unattainable because of technical difficulties.

Hemodynamic data and glycolytic variable measurements were analyzed by 2-way analysis of variance, with group and time as the 2 factors. Where the F value indicated significance, differences were isolated by the StudentNewman-Keuls post hoc test. Compliance curves were fit with use of a Levenberg-Marquardt algorithm. Comparisons of compliance curve coefficients (after natural logarithmic transformation) and equilibrium volumes at 30 and $60 \mathrm{~min}-$ utes of reperfusion to baseline values were made after 2way analysis of variance, followed by the least significant difference test (using the analysis of variance error mean square term), comparing to a population mean equal to zero change.

For myofibrillar ATPase activity, data from the pCaATPase activity relation were fit to the equation $\mathrm{Y}=\mathrm{A}+[(\mathrm{B}$ - A $\left.) /\left(1+\left(10^{\mathrm{C}} / 10^{\mathrm{X}}\right)^{\mathrm{D}}\right)\right]$ using nonlinear regression analysis to derive minimal (A) and maximal (B) ATPase activities, the $\left[\mathrm{Ca}^{2+}\right]$ at half maximal ATPase activity $(\mathrm{C})$, and the Hill coefficient (D), which characterizes the cooperativity of the relation. In this equation, $\mathrm{Y}$ was equal to inorganic phosphate liberated by ATPase activity and $\mathrm{X}$ was the $\left[\mathrm{Ca}^{+2}\right]$. The $\mathrm{pCa}_{50}$ (the $\mathrm{pCa}$ at which half-maximal activity occurs) was determined by taking the $-\log$ [coefficient C]. A 1-way analysis of variance was used to test for differences between groups in minimal and maximal ATPase activities, $\mathrm{pCa}_{50}$, and the Hill coefficient after log transformation, when required. 
Table I. Peak $(+) d P / d t$ and coronary flow rates

\begin{tabular}{llcc}
\hline & & Group & SPT \\
\cline { 2 - 4 } Time & \multicolumn{1}{c}{ ST\#2 } & ADO & \\
\hline Peak (+)dP/dt (mm Hg/sec) & & & $2289 \pm 76$ \\
$\quad$ Precardioplegia & $2380 \pm 68$ & $2468 \pm 82$ & $1845 \pm 142$ \\
30-min reperfusion & $1789 \pm 120^{*}$ & $2256 \pm 78$ & $1482 \pm 77^{* \dagger}$ \\
60-min reperfusion & $1640 \pm 135^{* \dagger}$ & $1981 \pm 116^{*}$ & \\
Coronary flow (mL/min) & & & $15.8 \pm 0.9$ \\
$\quad$ Precardioplegia & $16.0 \pm 2.0$ & $15.5 \pm 1.8$ & $13.5 \pm 2.3$ \\
30-min reperfusion & $13.0 \pm 2.0$ & $11.8 \pm 0.8$ & $11.8 \pm 2.0$ \\
60-min reperfusion & $12.8 \pm 1.8$ & $10.2 \pm 0.7$ & \\
\hline
\end{tabular}

In the three groups that underwent cardioplegic arrest, there were significant decreases in peak (+)dP/dt during reperfusion compared with precardioplegic arrest values. In the ST\#2 $(n=7)$ and SPT $(n=7)$ groups, at 60 minutes of reperfusion the peak $(+) \mathrm{dP} / \mathrm{dt}$ was significantly less than at 60 minutes of reperfusion in the ADO group $(n=6)$. Coronary flow measured before cardioplegic arrest showed no significant differences among the three cardioplegia groups $(n=6$ each). After 30 and 60 minutes of reperfusion, there were no significant differences in any of the groups compared with precardioplegia values and there were no significant intergroup differences in coronary flow.

${ }^{*} P<.05$ compared with precardioplegia.

${ }^{\dagger} P<.05$ compared with ADO at 60 minutes of reperfusion.

Table II. Changes in compliance coefficients

\begin{tabular}{llrrr}
\hline Group & Time (min) & \multicolumn{1}{c}{$\Delta V_{o}$} & $\Delta \ln A$ & $\Delta \ln B$ \\
\hline ST\#2 & 30-min reperfusion & $15.30 \pm 2.60^{*}$ & $0.854 \pm 0.165^{*}$ & $-0.226 \pm 0.048$ \\
& 60-min reperfusion & $17.26 \pm 2.11^{*}$ & $0.574 \pm 0.300^{*}$ & $-0.102 \pm 0.164$ \\
ADO & 30-min reperfusion & $11.21 \pm 8.76^{*}$ & $-0.277 \pm 0.124$ & $0.194 \pm 0.137$ \\
& 60-min reperfusion & $9.44 \pm 6.17^{*}$ & $0.044 \pm 0.502$ & $0.143 \pm 0.301$ \\
SPT & 30-min reperfusion & $14.76 \pm 4.77^{*}$ & $0.654 \pm 0.586^{*}$ & $0.470 \pm 0.315^{*}$ \\
& 60-min reperfusion & $8.65 \pm 6.29^{*}$ & $0.181 \pm 0.450$ & $0.726 \pm 0.238^{*}$ \\
\hline
\end{tabular}

Effects of cardioplegic arrest and reperfusion in the three cardioplegia groups on the equilibrium volume $\left(V_{o}\right)$ and compliance coefficients. The equilibrium volume increased significantly after cardioplegic arrest at 30 and 60 minutes of reperfusion in all three groups that had undergone cardioplegic arrest. Compliance coefficients A and B are presented in their natural logarithmic converted form for comparison. In the ST\#2 group there was a significant increase in the A coefficient, but B was unchanged from precardioplegia values. In the adenosine-supplemented group there was no significant change in either A or B. In the SPT group, A was significantly increased at 30 minutes of reperfusion, but returned to a value no different from baseline by 60 minutes. However, the B coefficient in the SPT group was significantly increased at both times. For all groups, $\mathrm{n}=6$.

${ }^{*} P<.05$ compared with zero.

\section{Results}

Cardiac function. Systolic pressure is represented at 2 and $10 \mathrm{~mm} \mathrm{Hg}$ preload pressures (Fig 1). For the ST\#2 group, left ventricular systolic pressure was significantly decreased at 30 and 60 minutes of reperfusion after cardioplegic arrest, at both levels of preload. In the ADO group, in which the cardioplegic solution was supplemented with $100 \mu \mathrm{mol} / \mathrm{L}$ adenosine, there was no significant change in systolic pressure from baseline values $(P=.4)$, indicating attenuation of myocardial depression. These values were not significantly different from those observed in the group of hearts that did not undergo cardioplegic arrest (No CPL; data not shown). When the adenosine receptor blocker 8-p-sulfophenyltheophylline was added to the cardioplegic solution (SPT group), the maintenance of systolic function seen in the adenosine group was ablated, indicating that the salutary effects of adenosine were receptor mediated. When the systolic function in the SPT group was compared with that in the ST\#2 group, there were no significant differences $(P=.4)$, but the systolic recovery was consistently lower in the SPT group than in the ST\#2 group at all times and preload pressures. This suggested that the 8-p-sulfophenyltheophylline blocked not only the effects of the exogenous adenosine in the adenosine group, but also endogenous adenosine produced in the ST\#2 group.

Peak (+)dP/dt and coronary flow rates are shown in Table I. All three groups that had undergone cardioplegic arrest demonstrated significant depression of maximum $(+) \mathrm{dP} / \mathrm{dt}$ by 60 minutes of reperfusion. In the SPT group, peak (+)dP/dt was significantly lower at 60 minutes of reperfusion than in the ADO group at the same time, demonstrating blockade of the functional recovery seen in the ADO group. At 60 minutes of reperfusion the value of peak $(+) \mathrm{dP} / \mathrm{dt}$ was also higher 
Table III. Myofibrillar ATPase measurements

\begin{tabular}{lcccc}
\hline Group & $p C a_{50}$ & Hill & Minimum & Maximum \\
\hline ST\#2 $(\mathrm{n}=7)$ & $6.15 \pm 0.03$ & $2.12 \pm 0.08^{*}$ & $37.91 \pm 10.2$ & $148.30 \pm 17.7$ \\
ADO $(\mathrm{n}=6)$ & $6.12 \pm 0.04$ & $2.50 \pm 0.13$ & $35.97 \pm 6.7$ & $152.18 \pm 16.5$ \\
SPT $(\mathrm{n}=8$ & $6.10 \pm 0.03$ & $2.16 \pm 0.04^{*}$ & $26.90 \pm 3.1$ & $152.67 \pm 10.1$ \\
No CPL $(\mathrm{n}=6)$ & $6.03 \pm 0.06$ & $2.48 \pm 0.04$ & $29.10 \pm 3.7$ & $143.97 \pm 8.3$ \\
\hline
\end{tabular}

There were no differences between the groups with respect to calcium sensitivity $\left(\mathrm{pCa}_{50}\right)$ minimum, or maximum enzyme activity. Relative to the No CPL group, cooperative interaction (Hill coefficient) was significantly depressed in the ST\#2 and SPT groups, but not in the ADO group.

"Difference from No CPL value.

Table IV. Effluent lactate concentrations (in micromoles per liter)

\begin{tabular}{|c|c|c|c|c|c|c|}
\hline \multirow[b]{2}{*}{ Group } & \multirow[b]{2}{*}{ Precardioplegia } & \multicolumn{3}{|c|}{ Cardioplegic arrest } & \multicolumn{2}{|c|}{ Reperfusion } \\
\hline & & $30 \mathrm{~min}$ & $60 \mathrm{~min}$ & $90 \mathrm{~min}$ & $30 \mathrm{~min}$ & $60 \mathrm{~min}$ \\
\hline ST\#2 & $40 \pm 20$ & $240 \pm 150^{* \dagger}$ & $370 \pm 50^{*}$ & $100 \pm 70^{\dagger}$ & $70 \pm 20$ & $60 \pm 30$ \\
\hline $\mathrm{ADO}$ & $50 \pm 20$ & $480 \pm 200^{*}$ & $460 \pm 210^{*}$ & $570 \pm 260^{*}$ & $70 \pm 20$ & $100 \pm 40$ \\
\hline SPT & $50 \pm 40$ & $110 \pm 60^{\dagger \neq}$ & $120 \pm 70^{\dagger *}$ & $110 \pm 50^{\dagger}$ & $40 \pm 20$ & $90 \pm 70$ \\
\hline
\end{tabular}

Effluent lactate concentrations were significantly elevated in the ST\#2 group $(n=7)$ at 30 and 60 minutes of reperfusion compared with baseline but returned to precardioplegia values by 90 minutes of reperfusion. In the ADO group $(n=6)$ there was a significant increase in effluent lactate at all cardioplegic arrest time points and this was significantly greater than that in the ST\#2 group at 30 and 90 minutes of cardioplegic arrest. When adenosine receptors were blocked (SPT group; $\mathrm{n}=$ 7) there was no significant increase in effluent lactate during cardioplegic arrest, and values were significantly lower than in the ADO group at all times and significantly lower than values in ST\#2 hearts at 60 minutes.

"Significant difference from baseline $(P<.05)$

'Significant difference from $\mathrm{ADO}(P<.05)$.

${ }^{*}$ Significant difference from ST\#2 $(P<.05)$.

in the ADO group than in the ST\#2 group. For the three cardioplegia groups there were no significant changes in coronary flow after cardioplegic arrest and reperfusion as compared with baseline values, nor were there any significant differences between the groups $(P=.2)$.

When the left ventricular EDPVR was normalized for the equilibrium volume by the equation $\mathrm{Y}=\mathrm{A}$. $\left(\exp ^{\mathrm{B} \cdot \mathrm{Vn}_{-}}-1\right)$, changes in compliance were assessed. Fig 2 is a representative example of compliance curves from one heart in the ST\#2 group. Before cardioplegic arrest the values of the $\mathrm{A}$ and $\mathrm{B}$ coefficients were 22.817 and 1.869 , respectively. However, after $60 \mathrm{~min}-$ utes of reperfusion, coefficient A increased to 27.914 and $\mathrm{B}$ increased to 2.237. These changes in the $\mathrm{A}$ and $B$ coefficients translated to a decrease in compliance, or a shift in the compliance curves up and to the left. The equilibrium volume after reperfusion increased significantly from baseline in all three cardioplegia groups, indicating a shift in the left ventricular EDPVR to the right (Table II). Because the stress/strain relationship is monoexponential, natural logarithmic conversion of the coefficients was required for statistical comparison. Changes in the A and B coefficients are shown in Table II as their natural logarithmic converted form. In the ST\#2 group, the B coefficient was unchanged $(P=.6)$, but A was significantly increased during reperfusion compared with values before cardioplegic arrest. Thus there was a significant decrease in compliance in these hearts. In the ADO group, there was no significant change in either coefficient $(P=.7)$, indicating that compliance was unchanged in the ADO group after cardioplegic arrest and reperfusion. In the SPT group there was a significant increase in the A coefficient at 30 minutes of reperfusion and in the B coefficient during reperfusion at both times, indicating that the prevention of decreased compliance by adenosine supplementation was adenosine-receptor mediated.

Myofibrillar ATPase activity. The myofibrillar pCaATPase activity relation was assessed to determine whether the decrease in myofilament ATPase cooperativity previously demonstrated in our laboratory ${ }^{14}$ after cardioplegic arrest and reperfusion might be altered by the supplementation of adenosine in the cardioplegic solution. The $\mathrm{pCa}_{50}$ was used as a measure of myofibrillar $\mathrm{Ca}^{2+}$ sensitivity and the Hill coefficient was a measure of cooperative activation of the myofilaments. Data for myofibrillar ATPase activity are shown in Table III. Similar to results in our previous work, ${ }^{14}$ the Hill coefficient was significantly depressed in the ST\#2 group, relative to that in hearts that did not undergo cardioplegic arrest. No significant changes were observed in the other variables, in agreement with our previous 
work. ${ }^{14}$ In the ADO group, the Hill coefficient was not significantly depressed, demonstrating that adenosine supplementation of cardioplegic solution can prevent the changes in myofilament interactions associated with cardioplegic arrest. In the presence of 8-p-sulfophenyltheophylline (SPT group), the ability of adenosine to prevent the depression in myofilament cooperativity was absent, indicating that this was an adenosine-receptor-mediated phenomenon. Therefore it appears likely that one of the mechanisms of adenosine-mediated improvement in myocardial protection is secondary to effects on the myofilament interactions.

Glycolytic activity. Effluent lactate concentrations are shown in Table IV. Effluent lactate levels were significantly increased (10-fold) in the ST\#2 group hearts at 30 and 60 minutes of cardioplegic arrest, but returned to precardioplegia levels by 90 minutes. Hearts in the ADO group displayed a significant increase in effluent lactate levels at 30,60, and 90 minutes of cardioplegic arrest, compared with baseline levels, and significantly higher levels at 30 and 90 minutes than levels in ST\#2 hearts. This suggests that supplementation of cardioplegic solution with adenosine increases anaerobic glycolysis during the duration of cardioplegic arrest. When adenosine receptors were blocked with 8-p-sulfophenyltheophylline, neither the increase in effluent lactate seen in the ADO group nor that in the ST\#2 group during cardioplegic arrest was evident, confirming that this was an adenosine-receptor-mediated response. At 60 minutes of cardioplegic arrest, both the ADO and the ST\#2 groups had effluent lactate levels that were higher than that in the SPT group, but the ADO group and the ST\#2 group effluent levels were not significantly different. This intermediate response in the ST\#2 group implicates an important role of endogenous adenosine in myocardial protection during cardioplegic arrest.

\section{Discussion}

The results of this study demonstrate that adenosine supplementation of cardioplegic solution improves myocardial functional recovery, which concurs with results obtained by other investigators. ${ }^{3-5,17,18} \mathrm{In}$ regard to the cellular mechanism of the improved recovery attributable to adenosine supplementation of cardioplegic solution, our data support two primary conclusions. Adenosine, via a receptor-mediated action, prevented the depression in myofibrillar cooperative action seen after cardioplegic arrest and reperfusion. This is evidenced by prevention of a decrease in the Hill coefficient in the ADO group and the reversion to decreased cooperativity in the presence of an adenosine-receptor antagonist. Improvements in postcardioplegic function were also associated with adenosine-receptor-mediated increased anaerobic glycolysis during cardioplegic arrest. This is indicated by the increase in effluent lactate concentrations collected in the ADO group, an effect ablated with adenosinereceptor blockade.

Our finding that the addition of adenosine during cardioplegic arrest improves recovery of myocardial mechanical function concurs with observations by other investigators. ${ }^{3-5,17,18} \mathrm{We}$ observed improvements in systolic pressure generated in the ADO group relative to results in the other two cardioplegic groups. In fact, both the ST\#2 and the SPT groups had a significant decrease in systolic pressure generation, whereas the adenosine-supplemented group had no significant decrease. Hudspeth and coworkers ${ }^{3}$ demonstrated that supplementation of blood cardioplegic solution with adenosine led to improvements in systolic recovery. More specifically, they observed an attenuation in the decrease in the slope of the end-systolic pressure-volume relationship after cardioplegic arrest and reperfusion in the adenosine-supplemented group, which was not evident in their control group. The improvements in diastolic recovery seen in the adenosine-supplemented group also correlate with findings by other investigators. ${ }^{3,5,18}$ Bolling and associates ${ }^{18}$ demonstrated improved myocardial functional recovery after cardioplegic arrest and reperfusion with the supplementation of cardioplegic solution with either adenosine or 2deoxycoformycin, a noncompetitive inhibitor of adenosine deaminase, the enzyme responsible for the degradation of adenosine to inosine.

Much of the prior work examining changes in compliance in adenosine-supplemented cardioplegic solution did not account for changes in the equilibrium volume of the left ventricle. It is necessary to evaluate changes in equilibrium volume to assess the stress/strain relationship. Once ventricular volume is normalized for the equilibrium volume, the stress/strain relationship (compliance) can be characterized by a monoexponential function, which was first described by Glanz and Parmley. ${ }^{16}$ In the present study, there was a significant decrease (shift of the curve up and to the left) in compliance in the ST\#2 group, as indicated by an increase in coefficient A. However, in the adenosinetreated group these changes were not evident. In contrast, when the adenosine receptors were blocked with 8-p-sulfophenyltheophylline, the improvements in recovery of compliance attributable to adenosine were attenuated. Although these changes in diastolic function would contribute to improved ventricular filling 
during diastole, they might also lead to improvements in systolic function.

Prior work from our laboratory demonstrated specific alterations in biochemical interactions in the myofibrillar contractile apparatus as a result of cardioplegic arrest. ${ }^{14}$ In this study, the cooperative interaction (Hill coefficient) of the actin-myosin $\mathrm{Mg}^{+2}$ ATPase was decreased after cardioplegic arrest. This finding was consistent in groups that underwent 2 hours of cardioplegic arrest with St Thomas' Hospital No. 2 cardioplegic solution with or without reperfusion, indicating that the decreased myofilament cooperativity was a result of the cardioplegic arrest and not secondary to reperfusion injury. These alterations would contribute to both the systolic and diastolic dysfunction seen after cardioplegic arrest and reperfusion. Our current finding of an adenosine-receptor-mediated prevention of both the decreased cooperativity and the decreased systolic and diastolic function associated with cardioplegic arrest lends further credence to this hypothesis. The specific alterations in myofilaments responsible for these changes are still unknown. We have previously shown no evidence of protein degradation after cardioplegic arrest, ${ }^{14}$ as has been shown to occur in warm ischemia. ${ }^{19}$ The relative steepness of the activation of myofilament activity by $\mathrm{Ca}^{++}$is a complex function of protein-protein interactions within a functional unit between near-neighbor functional units. As such, the alterations that would be responsible for the changes we have found in cooperative activation are likely to be subtle. It is clear from previous work that minor changes in protein interactions among thin filament proteins may be amplified considerably in terms of functional changes. ${ }^{20}$

Adenosine has been shown to increase myocardial glucose uptake ${ }^{11-13}$ and glycolysis, ${ }^{8,21}$ and this response has been shown to be associated with functional protection during warm ischemia, ${ }^{7}$ ischemic preconditioning, ${ }^{22}$ and critical coronary stenosis. ${ }^{10}$ However, work by Ning and colleagues ${ }^{15}$ suggested that myocardial glycolysis might reach a temperature threshold during cardioplegic arrest. Increased glycolysis was associated with cardioprotection during cardioplegic arrest only when temperatures were equal to or greater than $30^{\circ} \mathrm{C}$; temperatures lower than this were not associated with increased glycolysis. However, we found that adenosine-supplemented cardioplegic solution was associated with receptor-mediated increased anaerobic glycolysis throughout cardioplegic arrest, as evidenced by increased effluent lactate levels. These findings corroborate those recently reported by Friehs and coworkers, ${ }^{23}$ who also demonstrated significant increases in total lactate production when hearts were arrested with adenosine-supplemented cardioplegic solution. In the present study, there was a response in the ST\#2 group intermediate to those in the ADO and SPT groups; effluent lactate levels were significantly increased at 30 and 60 minutes of cardioplegic arrest but returned to a value not different from baseline by 90 minutes. This intermediate response is most likely a result of endogenous adenosine production, because both this and the amplified response seen in the ADO group were lost in the presence of 8-p-sulfophenyltheophylline. Thus, as in normothermic settings, it appears that adenosine is capable of increasing anaerobic glycolysis in the setting of cold cardioplegic arrest. Because these changes in anaerobic glycolysis paralleled the changes seen in functional recovery among the three cardioplegia groups, it is likely that increased anaerobic glycolysis contributes to the improved myocardial protection attributed to the supplementation of cardioplegic solution with adenosine.

A number of cellular events probably contribute to the salutary effects of adenosine-supplemented cardioplegic solution, but only recently have studies begun to identify these mechanisms. The present study is the first to implicate improvements in myofilament interactions as a mechanism of the action of adenosine in this unique setting of hypothermic arrest, and the study confirms the importance of increased glycolytic activity in this setting as well. Further study to determine the specific cellular changes responsible for these effects may lead to methods of optimizing these mechanisms for use in cardiac operations.

\section{REFERENCES}

1. Leung J. Clinical evidence of myocardial stunning in patients undergoing CABG surgery. J Card Surg 1993;8:220-3.

2. Foker JE, Einziz S, Wang T. Adenosine metabolism and myocardial preservation. J Thorac Cardiovasc Surg 1980;80:506-16.

3. Hudspeth DA, Nakanishi K, Vinten-Johansen J, et al. Adenosine in blood cardioplegia prevents postischemic dysfunction in ischemically injured hearts. Ann Thorac Surg 1994;58:1637-44.

4. de Jong JW, van der Meer P, van Loon H, Owen P, Opie LH. Adenosine as adjunct to potassium cardioplegia: effect on function, energy metabolism, and electrophysiology. J Thorac Cardiovasc Surg 1990;100:445-54.

5. Bolling SF, Bies LE, Gallagher KP, Bove EL. Enhanced myocardial protection with adenosine. Ann Thorac Surg 1989;47:809-15.

6. Silverman NA, Kohler J, Feinberg H, Levitsky S. Beneficial metabolic effect of nucleoside augmentation on reperfusion injury following cardioplegic arrest. Chest 1983;83:787-92.

7. Lasley RD, Mentzer RM Jr. Adenosine improves recovery of postischemic myocardial function via an adenosine A1 receptor mechanism. Am J Physiol 1992;263:H1460-5.

8. Lasley RD, Mentzer RM Jr. Adenosine increases lactate release 
and delays onset of contracture during global low flow ischemia. Cardiovasc Res 1993;27:96-101.

9. Lasley RD, Mentzer RM Jr. Protective effects of adenosine in the reversibly injured heart. Ann Thorac Surg 1995;60:843-6.

10. Fang HK, Sturgeon C, Segil LJ, Ripper RL, Law WR. Cardiac contractile function during coronary stenosis in dogs: association of adenosine in glycolytic dependence. Am J Physiol 1997;272: H2195-203.

11. Law WR, McLane MP. Adrenergic, insulin, and work interactions with adenosine's effects on in situ myocardial glucose uptake. Cardiovasc Res 1996;31:691-8.

12. Law WR, Raymond RM. Adenosine potentiates myocardial glucose uptake in vivo. Am J Physiol 1988;254:H970-5.

13. Law WR, McLane MP. Adenosine enhances myocardial glucose uptake only in the presence of insulin. Metabolism 1991;40:947-52.

14. Powers FM, Fogelson BG, Sturgeon C, Solaro RJ, Law WR. Depressed myofilament cooperativity associated with post-cardioplegic myocardial depression. J Mol Cell Cardiol 1998;30: 1779-87.

15. Ning XH, Xu CS, Song YC, et al. Temperature threshold and modulation of energy metabolism in the cardioplegic arrested rabbit heart. Cryobiology 1998;36:2-11.

16. Glantz SA, Parmley WW. Factors which affect the diastolic pressure-volume curve. Circ Res 1978;42:171-80.
17. de Jong JW, van der Meer P, Owen P, Opie LH. Prevention and treatment of ischemic injury with nucleosides. Bratisl Lek Listy 1991;92:165-73.

18. Bolling SF, Bies LE, Bove EL, Gallagher KP. Augmenting intracellular adenosine improves myocardial recovery. J Thorac Cardiovasc Surg 1990;99:469-74.

19. Van Eyk JE, Powers FM, Law WR, Larue C, Hodges RS, Solaro RJ. Breakdown and release of myofilament proteins during ischemia and ischemia/reperfusion in rat hearts: identification of degradation products and effects on the pCa-force relation [abstract]. Circ Res 1998;82:261-71.

20. Rarick HM, Opgenorth TJ, von Geldern TW, Solaro RJ. An essential myosin light chain peptide stimulates cardiac myofibrillar ATPase activity. J Biol Chem 1996;271:27039-43.

21. Wyatt DA, Ely SW, Lasley RD, et al. Purine-enriched asanguineous cardioplegia retards adenosine triphosphate degradation during ischemia and improves postischemic ventricular function. J Thorac Cardiovasc Surg 1989;97:771-8.

22. Janier MF, Vanoverschelde JLJ, Bergmann SR. Ischemic preconditioning stimulates anaerobic glycolysis in the isolated rabbit heart. Am J Physiol 1994;267:H1353-60.

23. Friehs I, Cao Danh H, Takahashi S, et al. Adenosine prevents protein kinase $\mathrm{C}$ activation during hypothermic ischemia. Circulation 1997;9:221-6.

\section{Timely}

The Journal of Thoracic and Cardiovascular Surgery delivers the information you need now. Articles usually appear within four months of acceptance. 